\title{
Forehead carbuncle with intractable headache
}

This article was published in the following Dove Press journal:

Neuropsychiatric Disease and Treatment

20 March 2015

Number of times this article has been viewed

\section{Ping-yin Chou',* \\ Yin-Chun Chen ${ }^{2, *}$ \\ Poyin Huang ${ }^{3,4}$}

'Department of Neurology, Kaohsiung Medical University Hospital,

Kaohsiung Medical University, Kaohsiung, Taiwan; ${ }^{2}$ Department of Dermatology, Kaohsiung Municipal Ta-Tung Hospital, Kaohsiung Medical University Hospital, Kaohsiung Medical University, Kaohsiung, Taiwan; ${ }^{3}$ Department of Neurology, Kaohsiung Municipal Hsiao-Kang Hospital, Kaohsiung Medical University Hospital, Kaohsiung Medical University, Kaohsiung, Taiwan; ${ }^{4}$ Department of Neurology, Faculty of Medicine, College of Medicine, Kaohsiung Medical University, Kaohsiung, Taiwan

*These authors contributed equally to this work
Correspondence: Poyin Huang Department of Neurology, Kaohsiung Medical University Hospital, Nol00, Tzyou Ist Road, Kaohsiung City 804, Taiwan

Tel +88673 I 2 I I 0 I ext 5945

Email total.control@msa.hinet.net
Abstract: Although carbuncles are commonly seen and may heal on their own or respond well to treatment, in rare conditions, bacteria from carbuncles can spread into the bloodstream and migrate to other areas of the body. Herein, we report on an elderly female who suffered from forehead carbuncle with intractable headache, later confirmed as having subgaleal abscess. Physicians should pay special attention to elderly and immune-compromised patients with carbuncles located on the middle of the face, especially when accompanied by intractable headache, to avoid poor outcome.

Keywords: carbuncle, headache, subgaleal abscess

\section{Introduction}

In rare conditions, bacteria from carbuncles can spread into the bloodstream and migrate to other areas of the body, causing serious complications such as septicemia and infections in the liver, bones, joints, heart, and central nervous system. We describe an original case of forehead carbuncle with intractable headache, later confirmed as a subgaleal abscess.

\section{Case history}

A 74-year-old female visited our hospital due to a $2 \mathrm{~cm}$, painful, erythematous nodule with a small necrotic plug on her forehead, which had been resident for 2 days. She also complained of mild headache and denied trauma history. She had suffered for 5 years from diabetes mellitus, which was not aggressively controlled. She denied other known medical or psychiatric history. A diagnosis of carbuncle was made, and incision and drainage were performed. Oral cephradine and acetaminophen were prescribed. One day later, the patient visited our emergency room again due to progressive erythema and worsening headache. She had mild fever $\left(37.7^{\circ} \mathrm{C}\right)$ and bilateral periorbital soft tissue swelling with local heat. Pus was noted oozing from the nodule. Laboratory data showed elevated white blood cell count $(10.52 \times 1,000$ cells $/ \mu \mathrm{L})$ and $\mathrm{C}$-reactive protein levels $(78.90 \mathrm{mg} / \mathrm{L})$. Preliminary diagnosis suspected cellulitis; she was admitted to Kaohsiung Municipal Ta-Tung Hospital, Kaohsiung, for further management. However, her headache kept worsening and interfered with her sleep over the next 2 days. She described the headache as severe, persistent aching all over her head, which was not associated with postural change. Nonsteroidal anti-inflammatory drugs and tramadol hydrochloride were administered but completely in vain. In the fear of central nervous system involvement, a neurologist was consulted. Thorough neurological examination revealed no specific findings. However, brain computed tomography (CT) was still arranged due to intractable headache. Brain CT showed no intracranial lesion, but subgaleal emphysema and abscess were suspected (Figure 1). A neurosurgeon was then consulted, and operative debridement was promptly arranged. During the operation, widespread subgaleal abscess was noted and drained. Cephalosporin was administered 


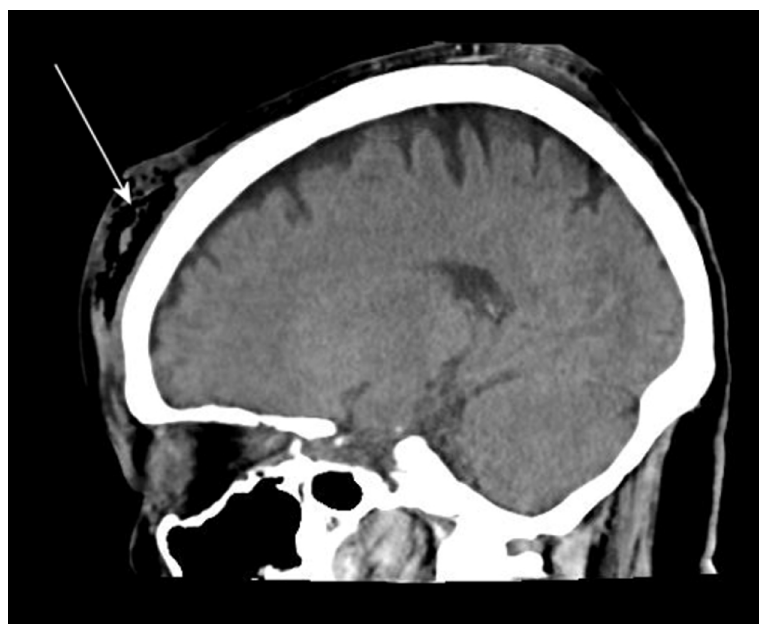

Figure I Subgaleal abscess in patient. Brain CT showed subcutaneous and subgaleal hypodensity indicating air and abscess (white arrow), later confirmed by operation. Abbreviation: CT, computed tomography.

according to the pus culture, which revealed Klebsiella pneumoniae. The patient improved gradually after treatment.

As previously described in the Case History section, this 74-year-old female had intractable headache, which developed in close relationship with subgaleal abscess. In addition, her headache resolved within 3 months after successful treatment of subgaleal abscess with operative debridement and antibiotics. According to the International Classification of Headache Disorders (ICHD-2) criteria, headache disorders attributed to extracranial infection of the head (such as ear, eye, and sinus infection) are coded as subtypes 11 . Headache or facial pain can be attributed to a disorder of the cranium, neck, eyes, ears, nose, sinuses, teeth, mouth, or other facial or cranial structures. ${ }^{1}$

\section{Discussion}

Carbuncle is a common dermatologic disease, and Staphylococcus aureus is the pathogen responsible in most cases. Though some authors advocate that systemic antimicrobial treatment is not needed for simple furuncles and carbuncles, ${ }^{2}$ we agree that incision and drainage with ancillary antimicrobial therapy is recommended for patients with immunosuppression or comorbidities, extremes of age; rapid progression to cellulitis and the lack of an adequate response to incision and drainage are also indications for such treatment. ${ }^{3}$ However, empiric antibiotics for carbuncle, even vancomycin, did not work for the unusual bacterial culture of Klebsiella in this patient. Although Klebsiella is strongly associated with infections in patients with diabetes, skin and soft tissue infections from Klebsiella are still uncommon. ${ }^{4}$ This case reminds us that empiric, systemic antibiotics for carbuncles and cellulitis may respond poorly in situations as described in the "Case history" section.

Subgaleal abscess is a rare, infectious complication. It is frequently associated with extended morbidity, and requires surgical intervention. The most common cause of subgaleal abscess is direct inoculation of microbes into the subgaleal space following scalp trauma. However, subgaleal abscess may result from hematogenous infection or contiguous spread, and the diagnosis may not be initially obvious. ${ }^{5}$ Carbuncles may progress to cellulitis and cause redness of the skin, swelling, and pain. Thus, headache in patients with cellulitis affecting face and scalp seems not unusual. However, carbuncles located on the middle of the face may raise concern, especially when the patient is elderly and immune-compromised. Intractable headache that is unresponsive to the standard medications and therapies utilized in the treatment of headaches also indicates the need for further survey. Head CT is often needed in the diagnosis of subgaleal abscess, and if operative debridement is not promptly performed, subgaleal abscess may further progress to life-threatening septicemia, osteomyelitis, and even subdural or brain abscess or meningitis. ${ }^{6}$ There are no focal neurological signs or specific symptoms in the early stages of subgaleal abscess. Intractable headache was the only "red flag" symptom in this patient. Thus, when encountering patients with intractable headaches, unusual causes should be kept in mind, which may include moyamoya syndrome, ${ }^{7}$ headache after botulinum A exotoxin injections, ${ }^{8}$ acquired immunodeficiency syndrome-related lymphoma confined to bone, ${ }^{9}$ migraine in obese individuals, ${ }^{10,11}$ and children with both migraine and periodic limb movement disorders in sleep. ${ }^{12}$ In conclusion, this case highlights that special attention should be paid to elderly and immune-compromised patients with carbuncles located on the middle of the face, especially when accompanied by intractable headache.

\section{Disclosure}

The authors report no conflicts of interest in this work.

\section{References}

1. Headache Classification Subcommittee of the International Headache Society. The International Classification of Headache Disorders: 2nd edition. Cephalalgia. 2004;24(Suppl 1):9-160.

2. Gorwitz RJ. The role of ancillary antimicrobial therapy for treatment of uncomplicated skin infections in the era of community-associated methicillin-resistant Staphylococcus aureus. Clin Infect Dis. 2007; 44(6):785-787.

3. Liu C, Bayer A, Cosgrove SE, et al. Clinical practice guidelines by the infectious diseases society of america for the treatment of methicillinresistant Staphylococcus aureus infections in adults and children. Clin Infect Dis. 2011;52(3):285-292. 
4. Joshi N, Caputo GM, Weitekamp MR, Karchmer AW. Infections in patients with diabetes mellitus. N Engl J Med. 1999;341(25):1906-1912.

5. Wiley JF 2nd, Sugarman JM, Bell LM. Subgaleal abscess: an unusual presentation. Ann Emerg Med. 1989;18(7):785-787.

6. Wang WH, Hwang TZ. Extensive subgaleal abscess and epidural empyema in a patient with acute frontal sinusitis. J Formos Med Assoc. 2003; 102(5):338-341.

7. Diaz UJ, Cabán-Martinez AJ, Halder GE. Presentation with recurrent intractable headache: a patient with moyamoya syndrome - case report. Neurol Med Chir (Tokyo). 2014;54(2):133-135.

8. Alam M, Arndt KA, Dover JS. Severe, intractable headache after injection with botulinum a exotoxin: report of 5 cases. $J$ Am Acad Dermatol. 2002;46(1):62-65.
9. Kelleher AD, Brew BJ, Milliken ST. Intractable headache as the presenting complaint of AIDS-related lymphoma confined to bone. J Acquir Immune Defic Syndr. 1994;7(6):629-630.

10. Verrotti A, Carotenuto M, Altieri L, et al. Migraine and obesity: metabolic parameters and response to a weight loss programme. Pediatr Obes. Epub 2014 Jul 3.

11. Verrotti A, Agostinelli S, D'Egidio C, et al. Impact of a weight loss program on migraine in obese adolescents. Eur J Neurol. 2013;20(2): 394-397.

12. Esposito M, Parisi P, Miano S, Carotenuto M. Migraine and periodic limb movement disorders in sleep in children: a preliminary case-control study. J Headache Pain. 2013;14:57.
Neuropsychiatric Disease and Treatment

\section{Publish your work in this journal}

Neuropsychiatric Disease and Treatment is an international, peerreviewed journal of clinical therapeutics and pharmacology focusing on concise rapid reporting of clinical or pre-clinical studies on a range of neuropsychiatric and neurological disorders. This journal is indexed on PubMed Central, the 'PsycINFO' database and CAS,

\section{Dovepress}

and is the official journal of The International Neuropsychiatric Association (INA). The manuscript management system is completely online and includes a very quick and fair peer-review system, which is all easy to use. Visit http://www.dovepress.com/testimonials.php to read real quotes from published authors.

Submit your manuscript here: http://www.dovepress.com/neuropsychiatric-disease-and-treatment-journal 